\title{
Relaxation Time Determinations by Progressive Saturation EPR: Effects of Molecular Motion and Zeeman Modulation for Spin Labels
}

\author{
V. A. Livshits, ${ }^{*} \dagger$ T. Páli, ${ }^{*}, 1$ and D. Marsh* \\ *Max-Planck-Institut für biophysikalische Chemie, Abteilung Spektroskopie, D-37070 Göttingen, Germany; and \\ $\dagger$ Centre of Photochemistry, Russian Academy of Sciences, 117421 Moscow, Russian Federation
}

Received April 28, 1997; revised January 13, 1998

The E PR spectra of nitroxide spin labels have been simulated as a function of microwave field, $H_{1}$, taking into account both magnetic field modulation and molecular rotation. It is found that the saturation of the second integral, $S$, of the first harmonic in-phase absorption spectrum is approximated by that predicted for slowpassage conditions, that is, $S \sim H_{1} / \sqrt{1+P H_{1}^{2}}$, in all cases. This result is independent of the degree of inhomogeneous broadening. In general, the fitting parameter, $P$, depends not only on the $T_{1}$ and $T_{2}$ relaxation times, but also on the rate of molecular reorientation and on the modulation frequency. Calibrations for determining the relaxation times are established from the simulations. For a given modulation frequency and molecular reorientation rate, the parameter obtained by fitting the saturation curves is given by $1 / P=a+1 / \gamma_{\mathrm{e}}^{2} T_{1} \cdot T_{2}^{\text {eff }}$, where $T_{2}^{\text {eff }}$ is the effective $T_{2}$. For molecular reorientation frequencies in the range $2 \times 10^{7}-2 \times 10^{8}$ $\mathrm{s}^{-1}, T_{2}^{\text {eff }}$ is dominated by the molecular dynamics and is only weakly dependent on the intrinsic $T_{2}^{0}$, allowing a direct estimation of $T_{1}$. For reorientation frequencies outside this range, the $\left(T_{1} T_{2}\right)$ product may be determined from the calibrations. The method is applied to determining relaxation times for spin labels undergoing different rates of rotational reorientation in a variety of environments, including those of biological relevance, and is verified experimentally by the relaxation rate enhancements induced by paramagnetic ions. $\odot 1998$ Academic Press

Key Words: progressive saturation E PR ; spin-lattice relaxation; spin labels; spectral simulation.

\section{INTRODUCTION}

As is well known, information on spin-lattice relaxation rates may be obtained from CW EPR spectra by performing progressive saturation experiments with increasing microwave power. Such methods have found increasing application in recent years with spin labels because of the structural information that may be derived by using paramagnetic relaxation agents (1-3) and also because of the sensitivity to a range of slow dynamic processes (4-6). Most of these applications have been studies with biological membranes. The method is particularly powerful in the general area of structural biology

\footnotetext{
${ }^{1}$ Permanent address: Institute of Biophysics, Biological Research Center, H-6701 Szeged, Hungary.
}

when it is combined with the newly introduced technique of site-directed spin labeling $(1,2)$.

Optimum application of the method is possible if absolute values for the relaxation times can be deduced directly from the saturation measurements. The principal problems involved in such determinations are the allowance for inhomogeneous broadening, the influence of rotational motion of the spin label, and the effects of magnetic field modulation. It has been proposed that the problems associated with inhomogeneous broadening may be circumvented by determining the saturation behavior of the second integral of the conventional in-phase first-harmonic absorption spectra (7). This analysis, however, was based on slowpassage conditions in the absence of molecular motion. The effects of rotational motion on the saturation behavior of the spectral lineheights have been analyzed by Haas et al. (8), but effects of the modulation field and inhomogeneous broadening were not explicitly considered. Passage effects associated with field modulation can be avoided by using low modulation frequencies, but it is desirable for practical reasons that these should be included explicitly in the analysis.

The aim of the present work is to develop an integrated approach to analysis of the EPR saturation curves of spin labels with specific allowance for both molecular rotational motion and Zeeman modulation effects, in a method that is not sensitive to inhomogeneous spectral broadening. This is done by using the uncorrelated jump model for the rotational motion (9) that readily may be incorporated in the Bloch equations including the microwave and modulation fields $(10,11)$. The advantage of this approach is that expressions for the lineshapes as a function of microwave power may be obtained in closed form. In the motional narrowing regime $\left(\tau_{\mathrm{R}}<10^{-9} \mathrm{~s}\right)$, however, the rotational contributions to the spin-spin relaxation rate from perturbation theory are incorporated directly in the Bloch equations. It is found that, under all conditions, the saturation curves for the doubleintegrated intensity of the conventional EPR spectra can be fitted by a functional dependence similar to that obtained for slowpassage conditions in the absence of molecular motion and that this is unaffected by inhomogeneous broadening. In general, values for the true $\left(T_{1} T_{2}\right)$ relaxation time product may be extracted from the saturation curves by using calibrations that are estab- 
lished for the simulations, over wide ranges of rotational correlation time. For frequencies of rotational motion in the intermediate range $\left(2 \times 10^{7}-2 \times 10^{8} \mathrm{~s}^{-1}\right)$, however, the saturation curves are relatively insensitive to the value of the intrinsic $T_{2}^{0}$, and the $T_{1}$ relaxation time itself (rather than just the combined $T_{1} T_{2}$ product) may be estimated directly. In the general case, values of the intrinsic $T_{2}^{0}$ that are needed to extract $T_{1}$ from the $\left(T_{1} T_{2}\right)$ product can be obtained from simulation of the low-field spectral components, with allowance for Gaussian inhomogeneous broadening. Relaxation times of spin labels in a variety of experimental systems have been obtained by using this approach, as well as spin-lattice relaxation rate enhancements that are induced by paramagnetic ions.

\section{MATERIALS AND METHODS}

The spin label TEMPOL (4-hydroxy-2,2,6,6-tetramethylpiperidine- $N$-oxyl) was obtained from Eastman Kodak (Rochester, NY), TEMPONE (4-oxo-2,2,6,6-tetramethylpiperidine- $N$ oxyl) from Aldrich (Milwaukee, WI), and TEMPO-stearate, TS (4-stearoyl-2,2,6,6-tetramethylpiperidine- $N$-oxyl), was from Molecular Probes (Eugene, OR). Spin-labeled phosphatidylcholines, $n$-PCSL (1-acyl-2-[n-(4,4-dimethyloxazolidine- $N$ oxyl)stearoyl]-sn-glycero-3-phosphocholine) with $n=5$ and $n=14$, were synthesized according to Ref. (12). Lysodecanoyl phosphatidylcholine, lyso PC (1-decanoyl-2-lyso-sn-glycero3-phosphocholine), was from Sigma (St. Louis, MO). Dioleoyl phosphatidylcholine, DOPC (1,2-dioleoyl-sn-glycero-3-phosphocholine), and dipalmitoyl phosphatidylcholine, DPPC (1,2dipalmitoyl-sn-glycero-3-phosphocholine), were from Avanti Polar Lipids (Alabaster, AL). The TEMPOL spin label was used at a concentration of $3 \times 10^{-4} \mathrm{M}$ (in glycerol-water solutions) and the spin-labeled lipids (in aqueous dispersions containing $1 \mathrm{mg}$ of total lipid) at a relative concentration of 1 $\mathrm{mol} \%$ for fluid lipid phases and $0.5 \mathrm{~mol} \%$ in lipid gel phases. All samples were saturated with argon.

EPR spectra were recorded on a 9-GHz Varian Century Line spectrometer at a magnetic field modulation frequency of $100 \mathrm{kHz}$ and over a scan range of 100 to $200 \mathrm{G}$, depending on the spectral extent at high microwave power. Samples of 5-mm length were contained in sealed 1-mm i.d. glass capillaries which were accommodated within a standard quartz EPR tube that contained light silicone oil for thermal stability. The samples were centred vertically in the microwave cavity. The entire sample chamber was sealed under argon. Temperature of the samples was controlled by nitrogen gas flow through a quartz dewar and measured by a fine-wire thermocouple located within the capillary in contact with the sample. Critical coupling conditions were used for the progressive saturation studies. The mean microwave field, $H_{1}$, at the sample was measured according to Ref. (13) and corrections for differences in cavity $Q$ were made as described in the same reference. Saturation transfer EPR spectra, where appropriate, were recorded according to the standardized protocol described in Ref. (14).
Spectra were simulated according to the methods described in the Theory section. Programs were written in Microsoft Quick-BASIC and numerical calculations were carried out on a $90-\mathrm{MHz}$ Pentium computer. Principal values used for the $g$ and hyperfine tensors were $\mathbf{g}=(2.0089,2.0061,2.0027)$ and $\mathbf{A}=(6.0 \mathrm{G}, 6.0 \mathrm{G}, 34.0 \mathrm{G})$, respectively. Simulated spectra were integrated twice over a magnetic field range of $200 \mathrm{G}$.

\section{THE ORY}

The Bloch equations that explicitly include the microwave and Zeeman modulation fields may be generalized to take into account molecular rotation by using the random jump diffusion model employed in Ref. (9). The resulting equations for the time dependence of the spin magnetization vector $\overrightarrow{\mathbf{X}}=(u, \mathrm{v}, z)$ in the rotating frame can be written in matrix form:

$$
\dot{\overrightarrow{\mathbf{X}}}+\left(\hat{\mathbf{A}}+f_{R} \hat{\mathbf{E}}\right) \cdot \overrightarrow{\mathbf{X}}=\overrightarrow{\mathbf{M}}_{O}+f_{R} \int \overrightarrow{\mathbf{X}} d \Omega,
$$

where $f_{R} \equiv \tau_{R}^{-1}$ is the frequency of isotropic rotational reorientation, $\overrightarrow{\mathbf{M}}_{o}=M_{o}\left(0,0, T_{1}^{-1}\right)$ and $\Omega \equiv(\theta, \varphi)$ is the orientation of the static magnetic field relative to the magnetic principal axes. The term containing $f_{R}$ on the left-hand side of Eq. [1] is the rate of transfer of spin magnetization to other orientations and that on the right-hand side is the rate of transfer from all other orientations. The Bloch equation matrix is given by

$$
\hat{\mathbf{A}}=\left(\begin{array}{ccc}
\left(T_{2}^{0}\right)^{-1} & \gamma_{e} d & 0 \\
-\gamma_{e} d & \left(T_{2}^{0}\right)^{-1} & -\gamma_{e} H_{1} \\
0 & \gamma_{e} H_{1} & T_{1}^{-1}
\end{array}\right),
$$

with $\gamma_{e}$ the electron gyromagnetic ratio and

$$
d=H-H_{r e s}(\theta, \varphi)+H_{m} \cos \omega_{m} t,
$$

where $H_{\text {res }}$ is the resonant field, $H_{\mathrm{m}}$ is the $p-p$ field modulation amplitude, and $f_{m}=\omega_{m} / 2 \pi$ is the modulation frequency. The resonant field is angular dependent being determined by the $\mathrm{g}$ and hyperfine tensor anisotropies:

$$
H_{r e s}(\theta, \varphi)=\frac{\hbar \omega_{e}}{\beta_{e} g(\theta, \varphi)}-m_{I} A(\theta, \varphi),
$$

where $\omega_{e}$ is the electron Larmor frequency and the tensor anisotropies, relative to the principal components, are given by

$$
\begin{gathered}
g(\theta, \varphi)=g_{z z} \cos ^{2} \theta+g_{x x} \sin ^{2} \theta \cos ^{2} \varphi+g_{y y} \sin ^{2} \theta \sin ^{2} \varphi \\
A(\theta, \varphi)=\left(A_{z z}^{2} \cos ^{2} \theta+A_{x x}^{2} \sin ^{2} \theta \cos ^{2} \varphi\right. \\
\left.+A_{y y}^{2} \sin ^{2} \theta \sin ^{2} \varphi\right)^{1 / 2} .
\end{gathered}
$$


Because of the periodic modulation field, the magnetization components $(u, \nu, z)$ are expanded in Fourier harmonics of $\omega_{m}$,

$$
u=\sum_{n=-\infty}^{\infty} u_{n} e^{-i n \omega_{m} t} ; \nu=\sum_{n=-\infty}^{\infty} \nu_{n} e^{-i n \omega_{m} t} ; z=\sum_{n=-\infty}^{\infty} z_{n} e^{-i n \omega_{m} t}
$$

where $u_{n}, \nu_{n}, z_{n}$ are the complex Fourier amplitudes.

Substituting Eqs. [7] into Eq. [1] gives an infinite system of coupled equations for the amplitudes $\overrightarrow{\mathbf{X}}_{n}=\left(u_{n}, \nu_{n}, z_{n}\right)$ :

$$
\begin{aligned}
& \hat{\mathbf{A}}_{0} \cdot \overrightarrow{\mathbf{X}}_{0}=\overrightarrow{\mathbf{M}}_{0}+f_{R} \int \overrightarrow{\mathbf{X}}_{0} d \Omega+\frac{1}{2} \hat{\gamma} \cdot H_{m}\left(\overrightarrow{\mathbf{X}}_{1}+\overrightarrow{\mathbf{X}}_{-1}\right) \\
& \hat{\mathbf{A}}_{n} \cdot \overrightarrow{\mathbf{X}}_{n}=f_{R} \int \overrightarrow{\mathbf{X}}_{n} d \Omega+\frac{1}{2} \hat{\gamma} \cdot H_{m}\left(\overrightarrow{\mathbf{X}}_{n+1}+\overrightarrow{\mathbf{X}}_{n-1}\right)
\end{aligned}
$$

with

$$
\hat{\mathbf{A}}_{n}=\hat{\mathbf{A}}^{\prime}+\left(f_{R}-i n \omega_{m}\right) \hat{\mathbf{E}}
$$

where $\hat{\mathbf{A}}^{\prime}$ is the Bloch matrix without the modulation field, which is now included explicitly in the term that contains the matrix:

$$
\hat{\gamma}=\left(\begin{array}{ccc}
0 & -\gamma_{e} & 0 \\
\gamma_{e} & 0 & 0 \\
0 & 0 & 0
\end{array}\right)
$$

Halbach (15) has shown that, in the small modulation amplitude approximation $\left(h_{m}=\gamma_{e} H_{m} T_{2}^{0}<1\right)$, the solution for a similar system (in the absence of diffusion terms) can be obtained by expanding the Fourier coefficients $u_{n}, \nu_{n}, z_{n}$ in a power series of the dimensionless modulation amplitude, $h_{m}$ :

$$
u_{n}=\sum_{\nu=0}^{\infty} u_{n, \nu} h_{m}^{\nu} ; \nu_{n}=\sum_{\nu=0}^{\infty} \nu_{n, \nu} h_{m}^{\nu} ; z_{n}=\sum_{\nu=0}^{\infty} z_{n, \nu} h_{m}^{\nu}
$$

The coefficients $u_{n, \nu}, \nu_{n, v}, z_{n, \nu}$ with $\nu<|n|$ disappear because of the symmetry properties of the Bloch equations. This result is readily shown to be valid for the present system as well, with molecular rotational diffusion terms included. It will be noted that the expansion given by Eq. [11] groups together the contributions from all intermodulation sidebands that depend upon a given power of $h_{m}$ (cf. Ref. 16). The separation of these sidebands $(100 \mathrm{kHz})$ is much less than the intrinsic linewidths (2-9 MHz).

Restriction to small modulation amplitudes $\left(h_{m}=\gamma_{e} H_{m} T_{2}^{0}\right.$ $<1$ ) means that only terms up to $\nu=1$ (and $n=1$ ) need to be retained in Eqs. [7], [11] for the first harmonic signal. This condition requires that the modulation amplitude be sufficiently low that the intensity of the first harmonic signal is linearly dependent on $H_{m}$. This criterion is readily established experimentally and corresponds to normal practice in recording first harmonic EPR spectra. (Preliminary simulations, using a method valid for all values of $H_{m}$, indicate that the saturation behavior of the integrated EPR signal is valid even up to $h_{m} \approx$ 1.5 , in spite of modulation broadening.) The equations for the vector coefficients of $\overrightarrow{\mathbf{X}}_{1,1}=\left(u_{1,1}, \nu_{1,1}, z_{1,1}\right)$ that define the magnetization components that are linear in $h_{m}$, and the equations for the zero-order coefficients of $\overrightarrow{\mathbf{X}}_{0,0}=\left(u_{0,0}, \nu_{0,0}, z_{0,0}\right)$ that correspond to the components independent of $h_{m}$, then can be written in the form

$$
\begin{aligned}
& \hat{\mathbf{A}}_{0} \cdot \overrightarrow{\mathbf{X}}_{0,0}=\overrightarrow{\mathbf{M}}_{o}+f_{R} \int \overrightarrow{\mathbf{X}}_{0,0} d \Omega \\
& \hat{\mathbf{A}}_{1} \cdot \overrightarrow{\mathbf{X}}_{1,1}=f_{R} \int \overrightarrow{\mathbf{X}}_{1,1} d \Omega+\left(\hat{\gamma} / \gamma_{e}\right) \cdot \overrightarrow{\mathbf{X}}_{0,0},
\end{aligned}
$$

where $f_{R}$ and the matrix elements of $\hat{\mathbf{A}}_{0}$ and $\hat{\mathbf{A}}_{1}$ are now dimensionless, which is obtained by multiplying their initial values by $T_{2}^{0}$. The solution of these integral equations [12] and [13] is relatively straightforward because the integrals $\overrightarrow{\mathbf{I}}_{0}=\int \overrightarrow{\mathbf{X}}_{0,0} d \Omega$ and $\overrightarrow{\mathbf{I}}_{1}=\int \overrightarrow{\mathbf{X}}_{1,1} d \Omega$ do not depend on the angular variables, $\Omega \equiv(\theta, \varphi)$. Thus, solving Eq. [12] for $\overrightarrow{\mathbf{X}}_{0,0}$ and integrating over $d \Omega$, one obtains

$$
\overrightarrow{\mathbf{I}}_{0}=\left(\hat{\mathbf{E}}-\hat{\mathbf{J}}_{0} f_{R}\right)^{-1} \cdot \hat{\mathbf{J}}_{0} \cdot \overrightarrow{\mathbf{M}}_{o}
$$

where $\hat{\mathbf{J}}_{0}=\int \hat{\mathbf{A}}_{0}^{-1} d \Omega$. Substituting from Eq. [14] in Eq. [12] then gives the solution:

$$
\overrightarrow{\mathbf{X}}_{0,0}=\hat{\mathbf{A}}_{0}^{-1} \cdot\left[\overrightarrow{\mathbf{M}}_{o}+f_{R}\left(\hat{\mathbf{E}}-\hat{\mathbf{J}}_{0} f_{R}\right)^{-1} \cdot \hat{\mathbf{J}}_{0} \cdot \overrightarrow{\mathbf{M}}_{o}\right]
$$

Similarly, solving Eq. [13] for $\overrightarrow{\mathbf{X}}_{1,1}$ and integrating over $d \Omega$ gives

$$
\overrightarrow{\mathbf{I}}_{1}=\left(\hat{\mathbf{E}}-\hat{\mathbf{J}}_{1} f_{R}\right)^{-1} \cdot \overrightarrow{\mathbf{J}}_{10}
$$

where $\hat{\mathbf{J}}_{1}=\int \hat{\mathbf{A}}_{1}^{-1} d \Omega$ and $\overrightarrow{\mathbf{J}}_{10}=\int \hat{\mathbf{A}}_{1}^{-1} \cdot \hat{\gamma} \cdot \mathbf{X}_{0,0} d \Omega$. The solution for $\overrightarrow{\mathbf{X}}_{0,0}$ in Eq. [15] is then used for evaluating $\overrightarrow{\mathbf{J}}_{10}$.

The lineshape of the first harmonic in-phase absorption spectrum is finally given by

$$
V_{1}=\operatorname{Re}\left\{V_{1,1}\right\}
$$

where $\overrightarrow{\mathbf{I}}_{1}=\left(U_{1,1}, V_{1,1}, Z_{1,1}\right)$. This result for the lineshape is obtained in closed form and requires only numerical evaluation of the integrals. The inhomogeneous line broadening due to hyperfine interaction with methyl and environmental protons 
can then be taken into account by convoluting this lineshape with a Gaussian function.

The use of the hyperfine resonance field (Eq. [4]) in the Bloch equations is an adiabatic approximation and therefore not applicable in the fast motional regime $\left(\tau_{R}<3 \times 10^{-9} \mathrm{~s}\right)$ (17). In this regime, rotational motion gives rise to a three-line spectrum and to an additional contribution to the spin-spin relaxation that is caused by rotational modulation of the hyperfine and Zeeman anisotropies. The contribution to the spinspin relaxation rates of the different hyperfine manifolds, $m_{l}$, contributed by this mechanism can be obtained from timedependent perturbation theory (18):

$$
T_{2}^{-1}\left(m_{I}\right)=T_{2,0}^{-1}+A+B m_{I}+C m_{I}^{2} .
$$

Here, $T_{2,0}^{-1}$ contains the residual homogeneous broadening arising from mechanisms other than molecular rotation. The coefficients $\mathrm{A}, \mathrm{B}$, and $\mathrm{C}$, which describe the motional broadening in Eq. [18], are directly proportional to the isotropic rotational correlation time $\left(\tau_{R} \equiv 1 / f_{R}\right)$ and are given by $(18)$

$$
\begin{aligned}
A= & \frac{4}{45}\left[(\Delta \omega)^{2}+3(\delta \omega)^{2}\right] \tau_{R}\left(1+\frac{3}{4} \frac{1}{1+\omega_{e}^{2} \tau_{R}^{2}}\right) \\
& +\frac{7}{45}\left[(\Delta A)^{2}+3(\delta A)^{2}\right] \tau_{R}\left(\frac{1}{1+\omega_{e}^{2} \tau_{R}^{2}}+\frac{3}{7} \frac{1}{1+\omega_{n}^{2} \tau_{R}^{2}}\right) \\
B= & \frac{8}{45}[\Delta \omega \Delta A+3 \delta \omega \delta A] \tau_{R}\left(1+\frac{3}{4} \frac{1}{1+\omega_{e}^{2} \tau_{R}^{2}}\right) \\
C= & \frac{4}{45}\left[(\Delta A)^{2}+3(\delta A)^{2}\right] \tau_{R} \\
& \times\left(1-\frac{1}{8} \frac{1}{1+\omega_{e}^{2} \tau_{R}^{2}}-\frac{3}{8} \frac{1}{1+\omega_{n}^{2} \tau_{R}^{2}}\right),
\end{aligned}
$$

where the tensor anisotropies $\Delta \omega=\left[g_{z z}-\frac{1}{2}\left(g_{x x}+g_{y y}\right)\right] \beta_{\mathrm{e}} / \hbar$ $H_{o}, \delta \omega=\frac{1}{2}\left(\mathrm{~g}_{\mathrm{xx}}-g_{y y}\right) \beta_{\mathrm{e}} / \hbar H_{o}, \Delta A=A_{z z}-\frac{1}{2}\left(A_{x x}+A_{y y}\right)$ and $\delta \mathrm{A}=\frac{1}{2}\left(A_{x x}-A_{y y}\right)$ are in angular frequency units, and $\omega_{\mathrm{n}} \approx \frac{1}{2}$ $a_{\mathrm{N}}$ is the nuclear Larmor frequency.

For the fast motional regime, spectra were simulated by omitting the terms containing $f_{R}$ in Eq. [1] and including instead the motional contributions to the spin-spin relaxation rate, $T_{2}^{-1}\left(m_{I}\right)$, from Eqs. [18]-[21] directly in the Bloch equation matrix (i.e., in Eq. [2]). For a direct comparison of the two methods, in the fast motional regime, it is necessary to omit the terms involving nuclear relaxation from Eqs. [19]-[21]. Then it is found that the power saturation curves calculated for the double-integrated intensities by using the time-dependent perturbation theory are almost identical to those obtained by the adiabatic approach, despite significant differences in lineshape arising from the somewhat different motional models. For simplicity, therefore, the adiabatic approximation is

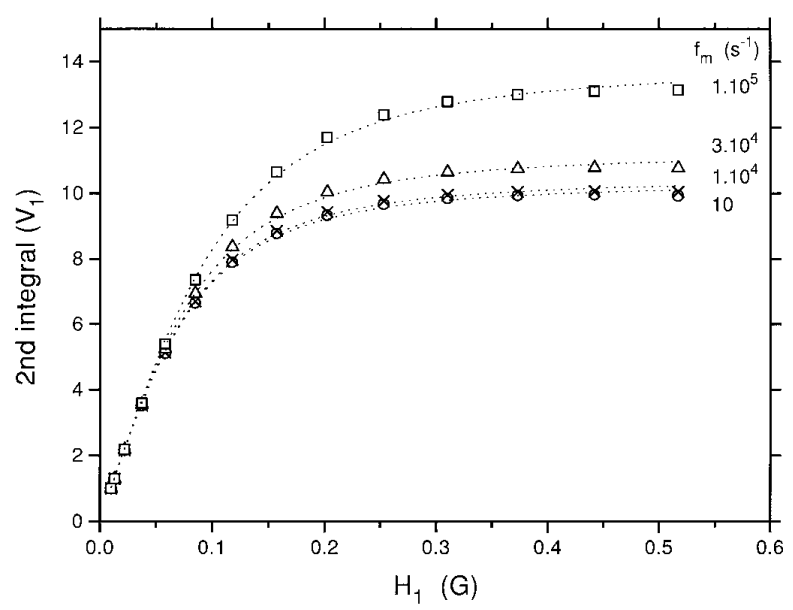

FIG. 1. Effects of magnetic field modulation frequency, $f_{m}$, on the microwave magnetic field $\left(H_{1}\right)$ dependence of the double integrated intensity of the first harmonic in-phase absorption EPR spectrum $\left(V_{1}\right)$ of nitroxide spin labels. Results are obtained from spectral simulations as described in the text, in the absence of molecular rotational motion. Simulated spectra were integrated numerically over a static magnetic field range of $H_{0}= \pm 100 \mathrm{G}$. Simulations are for intrinsic linewidth $\Delta H=1 \mathrm{G}$, spin-lattice relaxation time $T_{1}=5 \mu \mathrm{s}$, and the magnetic field modulation frequencies indicated (symbols). Dotted lines are nonlinear least-squares fits of the simulated data points to Eq. [22].

used throughout the whole rotational frequency range [cf. also Ref. (19)].

\section{RESULTS}

\section{Simulation of Saturation Curves}

The power saturation curves for the double-integrated intensity of the simulated in-phase first harmonic absorption spectra are given for different magnetic field modulation frequencies in Fig. 1. The saturation curves depend significantly on modulation frequency for $T_{1}$-relaxation times greater than 1-2 $\mu \mathrm{s}$ (Fig. 1). For $T_{1}=5 \mu \mathrm{s}$ and a spin-packet width of $1 \mathrm{G}$, the effective saturation factor decreases by about a factor of 2 at a modulation frequency of $100 \mathrm{kHz}$ compared with $10 \mathrm{kHz}$. For values of $T_{1}$ shorter than 1-2 $\mu \mathrm{s}$, the dependence on modulation frequency is small (data not shown). The saturation curves for higher modulation frequencies (Fig. 1) are, however, all similar in form to those obtained at low modulation frequency, which corresponds to true slow-passage conditions.

The dependence of the power saturation curves for the second integral of the simulated first harmonic absorption spectra on the frequency of molecular reorientation is given in Fig. 2. These results refer to a Zeeman modulation frequency of $100 \mathrm{kHz}$. As expected, sensitivity to rotational motion is found that corresponds to spectral diffusion of saturation in the slow-motional regime and to the conventional rotational contribution to the transverse relaxation rate in the faster motional regime. Nonetheless, the saturation curves all correspond quite 


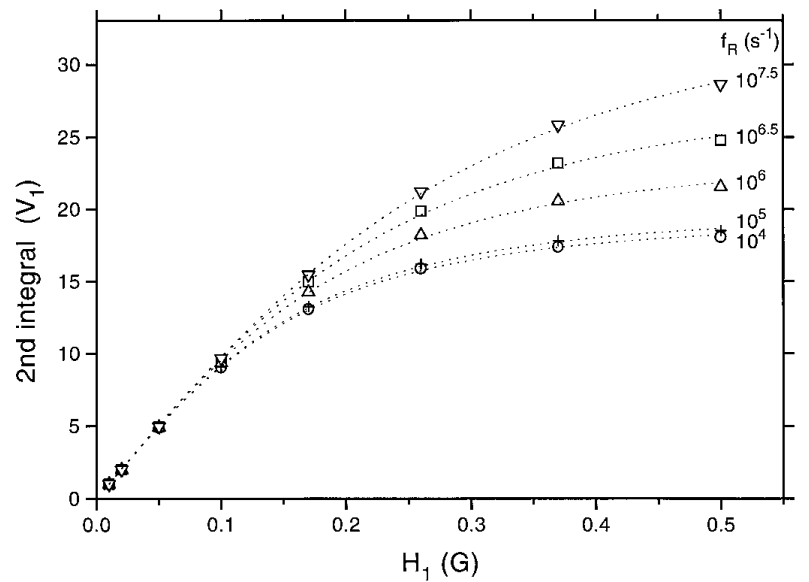

FIG. 2. Effects of molecular rotational frequency, $f_{R}$, on the microwave magnetic field $\left(H_{1}\right)$ dependence of the double integrated intensity of the first harmonic in-phase absorption EPR spectrum of nitroxide spin labels. Results are obtained from spectral simulations as described in the text, for $\Delta H=1 \mathrm{G}$, $T_{1}=1.8 \mu \mathrm{s}, \omega_{m} / 2 \pi=100 \mathrm{kHz}$, and the rotational frequencies indicated (symbols). The dotted lines are nonlinear least-squares fits of the simulated data points to Eq. [22].

closely to the form predicted for true slow-passage conditions in the absence of molecular reorientation.

Fitting simulated saturation curves. In spite of the sensitivity of the saturation curves both to molecular rotational reorientation and to the magnetic field modulation frequency, the dependence on the microwave magnetic field intensity, $H_{1}$, can be fitted satisfactorily by an expression of the form

$$
S\left(H_{1}\right) / S\left(H_{1,0}\right)=P_{1} H_{1} / \sqrt{1+P H_{1}^{2}}
$$

in all cases, where $P_{1}$ and $P$ are fitting parameters. This is exactly the form obtained for saturation of the second integral, $S\left(H_{1}\right)$, of the first-harmonic absorption spectrum with slowpassage conditions and in the absence of molecular motion. Under these latter conditions, the saturation parameter is given exactly by $P=\gamma_{e}^{2}\left(T_{1} T_{2}\right)$ and $P_{1}=1 / H_{1,0}$, where $\gamma_{e}$ is the electron gyromagnetic ratio and $H_{1,0}$ is a low microwave field intensity that corresponds to the absence of saturation.

Nonlinear least-squares fits of the saturation curves for the simulated spectra to Eq. [22] are given in Figs. 1 and 2, for different modulation frequencies and molecular rotational reorientation rates, respectively. As already stated, the form of the saturation curve is very similar to that predicted for slow-passage and "no-motion" conditions. The saturation curves for the double integrated spectral intensity are unchanged by the presence of inhomogeneous broadening (data not shown). This is found under all conditions and extends the generality of previous conclusions that were based on slow-passage analysis (7). The analytical expression for slow passage is therefore suitable for quantifying saturation curves of the double-integrated intensity of the first harmonic absorption spectra under essentially all experimental conditions. The value obtained for the fitting parameter $P_{1}$ is very close to $1 / H_{1,0}$ in all cases. The saturation curves therefore may be fitted with a single parameter, $P$, from which the relaxation times may be extracted. As seen, this parameter can depend sensitively on the experimental conditions. Calibrations to allow for the latter are thus necessary.

Extracting relaxation times. The values of the fitting parameter $P$, apart from their dependence on $T_{1}$ and $T_{2}^{0}$, depend substantially on the rotational correlation time (and Zeeman modulation frequency). However, the dependence of this parameter on the spin-lattice relaxation time, $T_{1}$, for each rotational frequency can be fitted satisfactorily by the simple dependence

$$
1 / P=a+b / T_{1}
$$

This relation is illustrated in Fig. 3 for simulations corresponding to different rotational frequencies and a modulation frequency of $100 \mathrm{kHz}$, where $a$ and $b$ are constants for a given rotational frequency and intrinsic $T_{2}$. The parameter $P$ is expressed here as an effective relaxation time product: $P / \gamma_{e}^{2}=$ $\left(T_{1} T_{2}\right)^{\text {eff }}$. Linearity is obtained in all cases, except for the critical region $f_{R} \sim 10^{7} \mathrm{~s}^{-1}$, where the relation becomes more approximate.

The parameter $b$ can be related to an effective $T_{2}$-value by $b$ $=1 / \gamma_{e}^{2} T_{2}^{e f f}$. This is an effective value because it comes from the saturation behavior of the spectrum integrated over all three hyperfine manifolds. The dependence of the effective transverse relaxation rate (i.e., $1 / T_{2}^{\text {eff }}$ ) on rotational frequency is given in Fig. 4 for two values of the intrinsic spin packet width, $\Delta H$. For slow rotational frequencies $\left(f_{R} \leq 10^{6} \mathrm{~s}^{-1}\right)$ and for fast rotational frequencies $\left(f_{R} \geq 10^{10} \mathrm{~s}^{-1}\right), 1 / T_{2}^{\text {eff }}$ is essentially

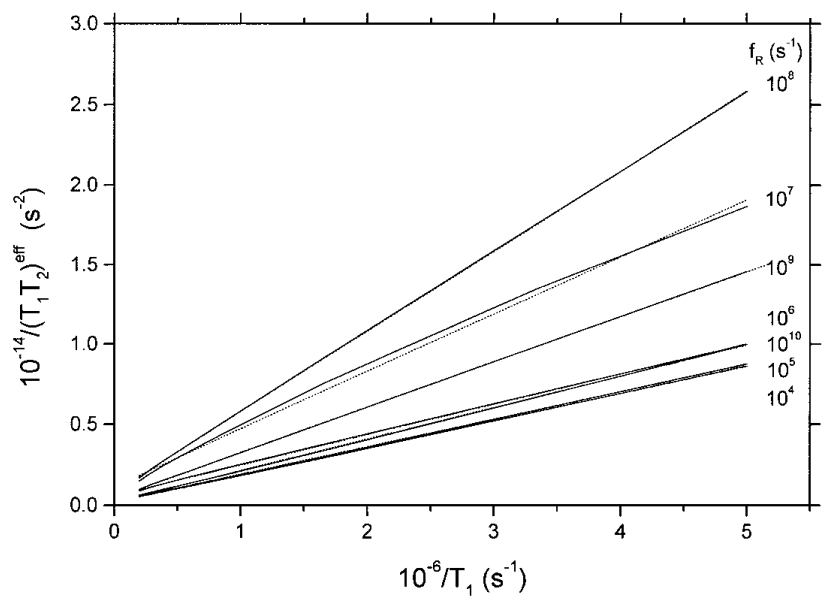

FIG. 3. Dependence of the reciprocal fitting parameter of the saturation curve, $1 / P \equiv 1 / \gamma_{e}^{2}\left(T_{1} T_{2}\right)^{\text {eff }}$, on the reciprocal spin-lattice relaxation time, $1 / T_{1}$, for different values of the molecular rotational frequency, $f_{R}$. Deduced from saturation curves (e.g., Fig. 2) for an intrinsic linewidth $\Delta H=1 \mathrm{G}$ and modulation frequency $\omega_{m} / 2 \pi=100 \mathrm{kHz}$. Dotted lines are linear regressions to Eq. [23]. 


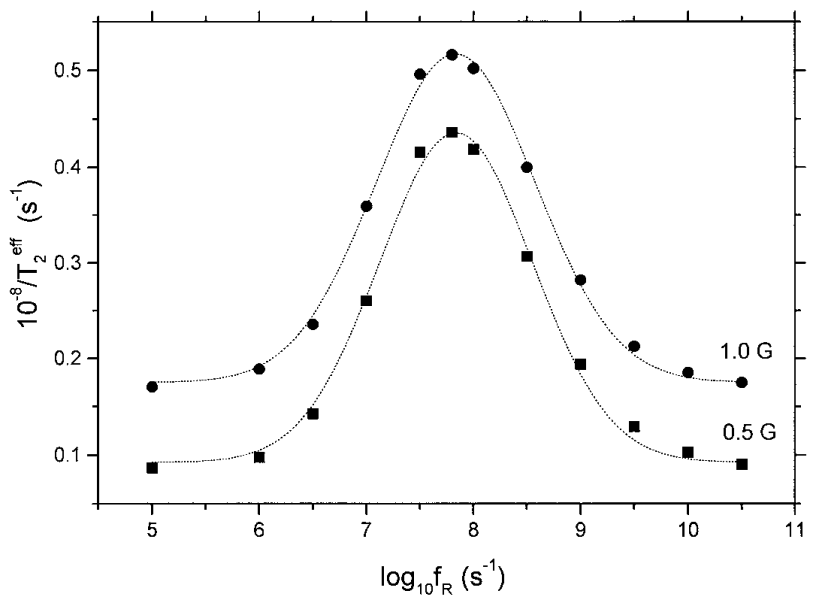

FIG. 4. Dependence of $1 / T_{2}^{\text {eff }}$, the effective $T_{2}$-relaxation rate, defined from the $b$ parameter in Eq. [23], on the molecular rotation frequency, $f_{R}$, deduced from the dependences of $P$ on $T_{1}$ that are given in Fig. 3. ( $\Delta H=0.5$ and $1 \mathrm{G}$, $T_{1}=0.2-5.1 \mu \mathrm{s}, \omega_{m} / 2 \pi=100 \mathrm{kHz}$.)

constant and equal to the intrinsic value, $1 / T_{2}^{0}$. In these two regions, the value of the parameter $a$ is also constant and close to zero. For intermediate rotational frequencies, $1 / T_{2}^{\text {eff }}$ becomes much greater with a maximum at approximately $f_{R} \sim 6.5 \times$ $10^{7} \mathrm{~s}^{-1}$. The increase in $1 / T_{2}^{\text {eff }}$ on the low-frequency side of the maximum corresponds to spectral diffusion arising from rotational reorientation (see, e.g., Ref. 20). The increase in $1 / T_{2}^{\text {eff }}$ on the high-frequency side of the maximum corresponds to conventional motional broadening arising from rotational modulation of the spectral anisotropies. Qualitatively, this behavior is reproduced experimentally by the saturation parameter measurements of Squier and Thomas (21), although the latter contain also the dependence of $T_{1}$ on rotational correlation time from other mechanisms (see Ref. 22).

As can be seen from Fig. 4, the positions and half-widths of the maxima in the dependence of $1 / T_{2}^{\text {eff }}$ on $\log f_{R}$, and the amplitudes of $1 / T_{2}^{\text {eff }}$ relative to the intrinsic relaxation rates, $1 / T_{2}^{0}$, are essentially the same for intrinsic spin packet widths differing by a factor of $2: \Delta H=0.5$ and $1 \mathrm{G}$. In fact, the effective transverse relaxation rate enhancements, $1 / T_{2}^{\text {eff }}-$ $1 / T_{2}^{0}$, decrease by not more than $10 \%$ when $\Delta H$ is increased from 0.5 to $2.0 \mathrm{G}$. This means that $1 / T_{2}^{\text {eff }}$ can be represented as a linear sum of an intrinsic and a rotationally induced spin-spin relaxation rate. The resulting calibration for $1 / T_{2}^{\text {eff }}$ in terms of rotational frequency can thus be parameterized numerically by the approximation

$$
1 / T_{2}^{e f f} \approx 1 / T_{2}+\left(5 \cdot 10^{7} / w\right) \exp \left[-\left(\log f_{R}-7.83\right)^{2} / w^{2}\right]
$$

where $w \approx 1.43-1.50$ depends only weakly on the intrinsic linewidth. Correspondingly, the calibration for the parameter $a$, defined in terms of $1 /\left(T_{1} T_{2}\right)^{\text {eff }}$, can be parameterized by

$$
\begin{aligned}
a /\left(10^{12} s^{2}\right) \approx & 8.9 \exp \left[-\left(\log f_{R}-6.9\right)^{2} / 0.7\right] \\
& +6.1 \exp \left[-\left(\log f_{R}-8.37\right)^{2} / 0.41\right]+1.6
\end{aligned}
$$

The effective excess spin-spin relaxation rate can also be described by a generalized spectral density (stretched exponential correlation function) similar to that given in Ref. (8), although with less numerical precision than the empirical expression that is given in Eq. [24].

In the intermediate region of rotational frequencies $\left(f_{R} \sim\right.$ $\left.2 \times 10^{7}-2 \times 10^{8} \mathrm{~s}^{-1}\right)$ corresponding to incipient motional narrowing, the rotational broadening of the spectrum is very significant. It can be considerably greater than the intrinsic linewidth, if the latter lies in the region of $0.5-1.0 \mathrm{G}$. For this intermediate range of rotational reorientation frequencies, the spin-lattice relaxation time can be estimated directly from the saturation curve, without detailed knowledge of the intrinsic $T_{2}^{0}$. This is illustrated in Fig. 5, which gives calibration curves for the spin-lattice relaxation time in terms of the fitting parameter $P$ (i.e., $\gamma_{e}^{2}\left(T_{1} T_{2}\right)^{\text {eff }}$ ) for different values of the intrinsic $T_{2}^{0}$ (or linewidth). The calibration curves for $\Delta H$ differing by a factor of 2 lie close together, indicating little sensitivity to the intrinsic $T_{2}^{0}$. The values of the linear fitting parameters in Eq. [23] for this range of rotational frequencies are given in Table 1. It is seen that these vary relatively little for different intrinsic linewidths, especially at rotational frequencies corresponding to the maximum in $1 / T_{2}^{\text {eff }}$.

For values of the rotational reorientation rate outside the critical intermediate range $f_{R} \sim 3 \times 10^{7}-3 \times 10^{8} \mathrm{~s}^{-1}$, the saturation fitting parameter, $P$, depends directly on the intrinsic $T_{2}^{0}$. The parameter that can be determined immediately from the saturation curves is then the $\left(T_{1} T_{2}\right)$ relaxation time product, as in the conventional "no-motion" case (cf. Ref. 7). This is

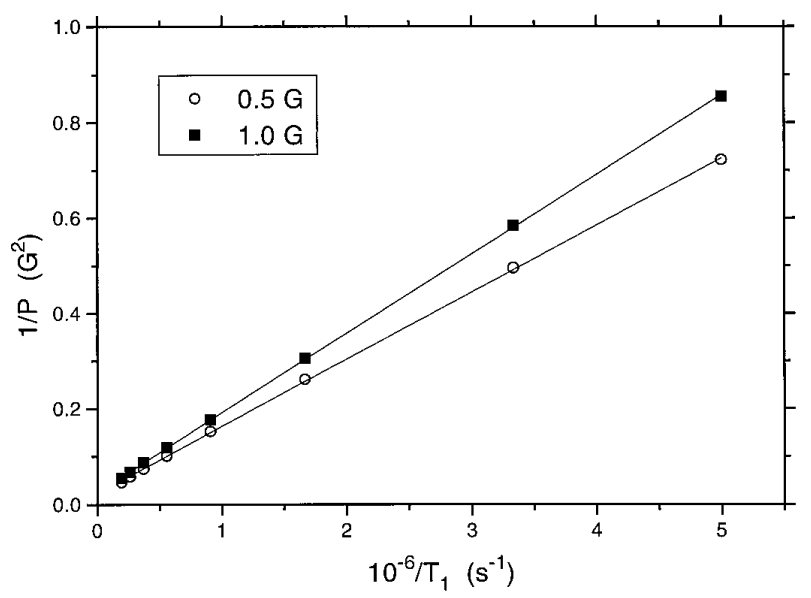

FIG. 5. Dependence of the reciprocal fitting parameter, $1 / P$, deduced from saturation curves, on the reciprocal spin-lattice relaxation time, $1 / T_{1}$, for a rotational frequency of $f_{R}=6 \times 10^{7} \mathrm{~s}^{-1}$, modulation frequency $\omega_{m} / 2 \pi=100$ $\mathrm{kHz}$, and different values of the intrinsic linewidth $\Delta H$ (i.e., $T_{2}^{0}$ relaxation time), as indicated. 
TABLE 1

Calibration of the Fitting Parameters $a$ and $b$ in Eq. [23] for Rotational Frequencies in the Range $10^{7} \mathrm{~s}^{-1}<f_{R}<10^{9} \mathrm{~s}^{-1}$ Which Give Rise to Extreme Motional Broadening

\begin{tabular}{lcccc}
\hline$f_{R}\left(\mathrm{~s}^{-1}\right)$ & $\Delta H(\mathrm{G})$ & $T_{1}(\mu \mathrm{s})$ & $a\left(\mathrm{G}^{2}\right)$ & $b \times 10^{-6}\left(\mathrm{G}^{2} \mathrm{~s}\right)$ \\
\hline $1.0 \times 10^{7}$ & $0.5-1.5$ & $(0.2-6.6)$ & $0.032-0.044$ & $0.083-0.145$ \\
$3.16 \times 10^{7}$ & $0.5-1.5$ & $(0.2-6.6)$ & $0.022-0.029$ & $0.137-0.185$ \\
$1.0 \times 10^{8}$ & $0.5-1.0$ & $(0.2-5.1)$ & $0.024-0.025$ & $0.134-0.162$ \\
$3.16 \times 10^{8}$ & $0.5-1.5$ & $(0.2-6.6)$ & $0.022-0.023$ & $0.100-0.129$ \\
$1.0 \times 10^{9}$ & $0.5-1.0$ & $(0.2-5.1)$ & $0.012-0.014$ & $0.063-0.091$ \\
\hline
\end{tabular}

Note. Results are given for different values of the intrinsic linewidth, $\Delta H$, and the range of $T_{1}$-values for which the fitting was performed is indicated. Zeeman modulation frequency: $\omega_{m} / 2 \pi=100 \mathrm{kHz}$.

illustrated by Fig. 6, in which the effective $1 /\left(T_{1} T_{2}\right)$ product deduced from fitting the simulated saturation curves is plotted against the true values used as input parameters for the simulations. Values of $T_{1}$ and intrinsic linewidth have been varied in different simulations over the ranges $0.2-5 \mu \mathrm{s}$ and $0.5-3.0$ $\mathrm{G}$, respectively. For rotational reorientation frequencies in the ranges $f_{R}<10^{6} \mathrm{~s}^{-1}$ and $f_{R}>3 \times 10^{9} \mathrm{~s}^{-1}$, the measured values of $1 /\left(T_{1} T_{2}\right)^{\text {eff }}$ are uniquely related to the true relaxation time product, irrespective of the individual values of $T_{1}$ and $T_{2}^{0}$. At the extremes, for values of $f_{R}=3 \times 10^{6} \mathrm{~s}^{-1}$ and $10^{9} \mathrm{~s}^{-1}$, this relation only holds approximately true, but the calibration curves lie within a sufficiently narrow region for the $\left(T_{1} T_{2}\right)$ product to be estimated with a tolerable degree of accuracy.

Fitting parameters, $a^{\prime}$ and $b^{\prime}$, for the $\left(T_{1} T_{2}\right)$ calibrations can be given in terms of the linear relation

$$
1 /\left(T_{1} T_{2}\right)^{e f f}=a^{\prime}+b^{\prime} /\left(T_{1} T_{2}\right) .
$$

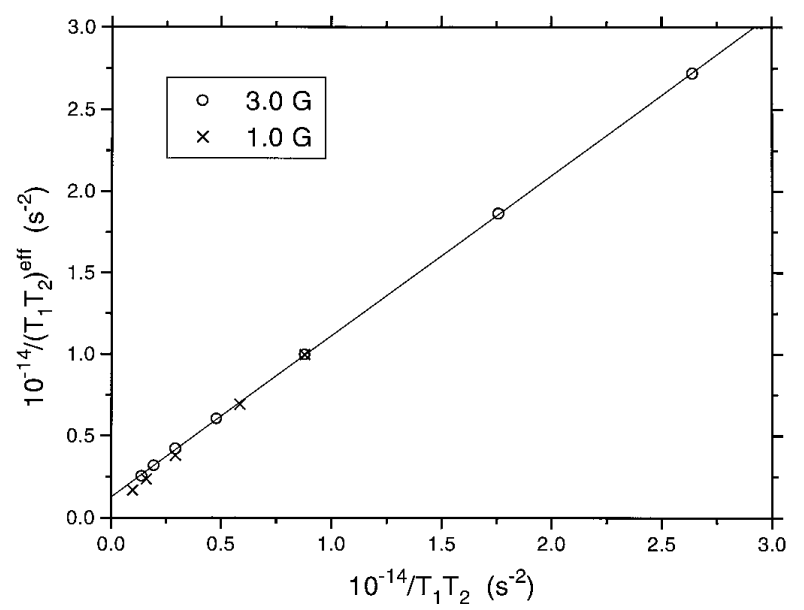

FIG. 6. Dependence of the reciprocal effective $\left(T_{1} T_{2}\right)$ relaxation time product, $\left(1 / T_{1} T_{2}\right)^{\text {eff }}$, deduced from the values of $P$ used in fitting the simulated saturation curves to Eq. [22] on the input value of the reciprocal relaxation time product, $1 /\left(T_{1} T_{2}\right)$, used for the simulations, for molecular rotation frequency $f_{R}$ $=10^{6} \mathrm{~s}^{-1}$, modulation frequency $\omega_{m} / 2 \pi=100 \mathrm{kHz}$, and values of $T_{1}$ in the range $0.2-4 \mu \mathrm{s}$ and of $\Delta H$ in the range $1.0-3.0 \mathrm{G}$, as indicated.
TABLE 2

Calibration of the Fitting Parameters $a^{\prime}$ and $b^{\prime}$ in Eq. [26] for R otational Frequencies in the Ranges $10^{5} \mathrm{~s}^{-1}<f_{R}<10^{7} \mathrm{~s}^{-1}$ and $10^{9} \mathrm{~s}^{-1}<f_{R}<3.16 \times 10^{10} \mathrm{~s}^{-1}$ for W hich R otational Broadening Is Not Appreciable

\begin{tabular}{llll}
\hline \multicolumn{1}{c}{$f_{R}\left(\mathrm{~s}^{-1}\right)$} & $\Delta H(\mathrm{G})$ & $a^{\prime} \times 10^{-14}\left(\mathrm{~s}^{-2}\right)$ & $b^{\prime}$ \\
\hline $1.0 \times 10^{5}$ & $1.0-3.0$ & $0.01-0.06$ & $0.97-0.93$ \\
$1.0 \times 10^{6}$ & $1.0-2.8$ & $0.037-0.11$ & $1.07-1.00$ \\
$3.16 \times 10^{6}$ & $1.0-3.0$ & $0.10-0.16$ & $1.34-1.10$ \\
$6.0 \times 10^{6}$ & $1.0-3.0$ & $0.115-0.164$ & $1.67-1.22$ \\
$1.0 \times 10^{7}$ & $1.0-3.0$ & $0.126-0.172$ & $2.03-1.33$ \\
$1.0 \times 10^{7}$ & $0.5-1.0$ & $0.090-0.126$ & $2.96-2.03$ \\
$1.0 \times 10^{9}$ & $0.5-1.0$ & $0.037-0.043$ & $2.20-1.60$ \\
$3.16 \times 10^{9}$ & $0.5-1.0$ & $0.016-0.025$ & $1.47-1.21$ \\
$1.0 \times 10^{10}$ & $0.5-1.0$ & $0.011-0.019$ & $1.17-1.05$ \\
$3.16 \times 10^{10}$ & $0.5-1.0$ & $0.009-0.018$ & $1.03-1.00$ \\
\hline
\end{tabular}

Note. Values are given for different intrinsic linewidths, $\Delta H$, and are fitted over the interval $T_{1}=0.2-5.1 \mu \mathrm{s}$. Zeeman modulation frequency: $\omega_{m} / 2 \pi=$ $100 \mathrm{kHz}$.

These calibration constants corresponding to the ranges of rotational frequency for which motional broadening is not appreciable are given in Table 2. Close to single, unique values are obtained for very fast and very slow motions, independent of the values of the intrinsic $T_{2}^{0}$ or linewidth. For these regions, the slope of the calibrations tends to unity and the intercept becomes very small. As the critical range of rotational frequency for extreme motional broadening is approached from either side, the calibration parameters then come to depend upon the value of the intrinsic linewidth. Under these conditions, the slope of the calibrations decreases towards unity with increasing spin packet width. For rotational frequencies $f_{R}=$ $10^{7} \mathrm{~s}^{-1}$ and $f_{R}=10^{9} \mathrm{~s}^{-1}$, the calibrations overlap with those given in Table 1 and neither approach yields unique values independent of $T_{2}^{0}$.

Depending on the rotational reorientation frequency, either the $\left(T_{1} T_{2}\right)$ relaxation time product or the spin-lattice relaxation time $T_{1}$ therefore may be determined from fitting the microwave power saturation curves in a continuous-wave progressive saturation experiment (Eq. [22]). This procedure requires an estimate of the rotational correlation time/reorientation frequency, which can be obtained from either the conventional or the saturation transfer EPR lineshapes, as appropriate, and then using the corresponding calibration curves (cf. Figs. 3-6). It should be noted that, although the simulated dependences of the saturation parameter on $1 / T_{1}$ or $1 /\left(T_{1} T_{2}\right)$ can be fitted satisfactorily by the linear equations [23] and [26] over a rather wide range of $T_{1}$-values, these dependences become nonlinear on extension to longer $T_{1}$-values. This nonlinearity is evidently due to the fact that the effects of rotation-induced diffusion and modulation frequency can only be taken into account approximately by an effective $T_{1}$ and $T_{2}$, especially at long $T_{1}$. Application of the methods is demonstrated in the following Experimental section, for spin-label systems with a range of 
TABLE 3

R elaxation Parameters Deduced from the Saturation Properties of TEMPOL in Glycerol-Water Mixtures of Different Viscosities

\begin{tabular}{|c|c|c|c|c|c|c|}
\hline $\begin{array}{c}\text { Glycerol } \\
(\%)\end{array}$ & $T\left({ }^{\circ} \mathrm{C}\right)$ & $\tau_{R}(\mathrm{~ns})$ & $\frac{10^{-14}}{\left(T_{1} T_{2}\right)^{e f f}}\left(\mathrm{~s}^{-2}\right)$ & $\Delta H(\mathrm{G})$ & $\begin{array}{c}\left(T_{1} T_{2}\right) \times 10^{14} \\
\left(\mathrm{~s}^{2}\right)\end{array}$ & $T_{1}(\mu \mathrm{s})$ \\
\hline 100 & -10 & 160 & $0.102 \pm 0.06$ & $1.65 \pm 0.05$ & $36.5 \pm 2.5$ & $10.6 \pm 0.7$ \\
\hline 90 & +5 & $8.5 \pm 0.5$ & $0.216 \pm 0.011$ & $1.95 \pm 0.05$ & $14.0 \pm 1.5$ & $4.8 \pm 0.6$ \\
\hline 70 & +15 & $0.120 \pm 0.005$ & $0.0815 \pm 0.002$ & - & $16.7 \pm 1.5$ & - \\
\hline
\end{tabular}

different rotational correlation times. For the correlation time regions which border between the extreme slow or fast motional regimes and the intermediate regime of strong motional broadening, a knowledge of $T_{2}^{0}$ is required to choose the appropriate calibration. This can be obtained from simulation of the low-power spectra, allowing for inhomogeneous broadening, as is illustrated in the Experimental section below.

\section{Experimental Saturation Curves}

Saturation curves were obtained for the small, soluble spin label TEMPOL in glycerol-water mixtures of varying viscosity and for spin-labeled phospholipids in various lipid and membrane environments that correspond to different rotational mobilities. In the latter case, the spin-lattice relaxation time, $T_{1}$, was also varied via the relaxation enhancement induced by paramagnetic ions. The saturation curves were constructed by evaluating the second integral of the in-phase first-harmonic absorption spectra which were fitted to Eq. [22] using values of $H_{1}$ at the sample. As found previously (7), the experimental saturation curves could be described well by Eq. [22], in all cases. All experiments were performed with a modulation frequency of $100 \mathrm{kHz}$, and calibrations were used from simulations that specifically took account of this Zeeman modulation. The modulation amplitude was restricted to $(1 / 3) \Delta H^{P P}$, or less, in order to fulfill the conditions used for the simulations. In addition, the modulation amplitude dependence was tested for the TEMPONE spin label in $80 \%$ aqueous glycerol at $20^{\circ} \mathrm{C}$. The saturation behavior of the integrated spectral intensity was found to be independent of the modulation amplitude over the range $H_{m}=(0.1-0.5) \times \Delta H^{P P}$. For the same sample it was also found that the $\left(T_{1} T_{2}\right)$ products obtained from the saturation curves for the three individual hyperfine lines were inversely proportional to their widths, that is, that the effective $T_{1}$ was the same, to within experimental error, for all three lines, as is assumed in fitting the integrals of the entire spectrum.

1. TEMPOL/glycerol-water mixtures. The viscosity of the solutions was adjusted by varying the glycerol content and the temperature to obtain rotational correlation times corresponding to the slow motional $\left(\tau_{R} \sim 1.6 \times 10^{-7} \mathrm{~s}\right)$, intermediate $\left(\tau_{R} \sim 10^{-8} \mathrm{~s}\right)$ and fast motional $\left(\tau_{R} \sim 10^{-10} \mathrm{~s}\right)$ regimes, as determined from the conventional spectral lineshapes in comparison with simulations (see also Ref. 23). These regimes correspond to the samples containing 100\%,
$90 \%$, and $70 \%$ glycerol, respectively, in Table 3 . The effective $\left(T_{1} T_{2}\right)$ products obtained from fitting the saturation curves to Eq. [22], and also the values of $T_{1}$ for the samples with $100 \%$ and $90 \%$ glycerol and of the true $\left(T_{1} T_{2}\right)$ relaxation time product for the sample with $70 \%$ glycerol, which are deduced from the calibrations obtained by simulation, are given in Table 3 . The sample with $100 \%$ glycerol lies on the edge of the intermediate motional regime where $T_{2}^{0}$ is required to select the correct calibration and hence to obtain a value for $T_{1}$. The value of $T_{2}$ was determined from lineshape simulations. This was also done for the sample with $90 \%$ glycerol to improve accuracy in the determination of $T_{1}$, although for samples in this intermediate motional regime a reasonable estimate of $T_{1}$ can be obtained without detailed knowledge of $T_{2}^{0}$. The values of the $\left(T_{1} T_{2}\right)$ product obtained from the separate measurements of $T_{1}$ and $T_{2}$ for these two samples are also reported in Table 3. It was found that the measured values of the saturation parameter for TEMPOL in glycerol-water mixtures studied with $100 \%$ and $70 \%$ glycerol exceeded those corresponding to the linear calibrations given in Tables 1 and 2 . Therefore, nonlinear calibrations obtained by simulation for longer $T_{1}$ intervals ( 1 to 10 or $15 \mu \mathrm{s}$ ) were used to extract values of $T_{1}$ or the $\left(T_{1} T_{2}\right)$ product from the experimental data, in these particular instances.

It is interesting to note that the extent of saturation (i.e., the value of $\left(T_{1} T_{2}\right)^{\text {eff }}$ ) for the fast-rotating spin label (in $70 \%$ glycerol) is somewhat greater than that for the slow-rotating spin label (in 100\% glycerol), although the latter was measured at a lower temperature. This difference is due to spectral diffusion for the slower rotating spin label. Correspondingly, the values of the $\left(T_{1} T_{2}\right)$ product deduced for these two systems are found to be in the reverse order to their effective values (see Table 3). This is consistent with the fast rotating spin label in $70 \%$ glycerol having a shorter $T_{1}$ than that for the slower rotating spin label in $100 \%$ glycerol.

2. Lipid spin labels in lysolipid micelles and fluid lipid bilayers. The EPR spectra of phosphatidylcholine spin-labeled at the 5-position of the chain (5-PCSL) in micelles of lysodecanoyl phosphatidylcholine at $34^{\circ} \mathrm{C}$ correspond to nearly isotropic (or weakly anisotropic) rotation because of averaging of the intramicellar motional anisotropy by the overall micelle rotation. The effective rotational correlation time estimated 
TABLE 4

Relaxation Parameters Deduced from the Saturation Properties of Spin-Labeled Phosphatidylcholine ( $n$-PC SL) and of TEMPO-stearate (TS) in Lipid Micelles and Fluid Lipid Bilayers

\begin{tabular}{|c|c|c|c|c|c|c|c|}
\hline Label & Lipid & $\begin{array}{c}{\left[\mathrm{Ni}^{2+}\right]} \\
(\mathrm{mM})\end{array}$ & $T\left({ }^{\circ} \mathrm{C}\right)$ & $\tau_{R}(\mathrm{~ns})$ & $\frac{10^{-14}}{\left(T_{1} T_{2}\right)^{e f f}}\left(\mathrm{~s}^{-2}\right)$ & $\Delta H(\mathrm{G})$ & $T_{1}(\mu \mathrm{s})$ \\
\hline 5-PCSL & LysoPC & 0 & 34 & $3.7 \pm 0.2$ & $0.97 \pm 0.05$ & $3.05 \pm 0.05$ & $0.85 \pm 0.07$ \\
\hline 14-PCSL & DOPC & 0 & 20 & $1.6 \pm 0.05$ & $1.05 \pm 0.05$ & $1.7 \pm 0.05$ & $0.44 \pm 0.02$ \\
\hline 14-PCSL & DOPC & 25 & 20 & $1.6 \pm 0.05$ & $1.16 \pm 0.05$ & $1.7 \pm 0.05$ & $0.41 \pm 0.02$ \\
\hline TS & DOPC & 0 & 20 & $1.5 \pm 0.05$ & $0.614 \pm 0.035$ & $0.57 \pm 0.03$ & $0.43 \pm 0.03$ \\
\hline TS & DOPC & 5 & 20 & $1.5 \pm 0.05$ & $0.67 \pm 0.04$ & $0.58 \pm 0.03$ & $0.39 \pm 0.03$ \\
\hline TS & DOPC & 10 & 20 & $1.5 \pm 0.05$ & $0.8 \pm 0.05$ & $0.61 \pm 0.03$ & $0.33 \pm 0.03$ \\
\hline TS & DOPC & 25 & 20 & $1.5 \pm 0.05$ & $1.27 \pm 0.06$ & $0.70 \pm 0.03$ & $0.22 \pm 0.02$ \\
\hline
\end{tabular}

from the ratios of the lineheights in the conventional EPR spectrum is $\tau_{R} \approx 3.7 \times 10^{-9} \mathrm{~s}$ (see, e.g., Ref. 18). The EPR spectra of phosphatidylcholine spin-labeled at the 14-position of chain (14-PCSL) in fluid bilayers of dioleoyl phosphatidylcholine (DOPC) at $20^{\circ} \mathrm{C}$ correspond to an effective rotational correlation time of $\tau_{R} \approx 1.6 \times 10^{-9} \mathrm{~s}$, although the spectra are somewhat anisotropic. The rotational correlation time of TEMPO-stearate in fluid DOPC bilayers is in the region of $1.5 \times 10^{-9} \mathrm{~s}$ at $20^{\circ} \mathrm{C}$, as evidenced by the conventional EPR spectral lineshapes. The correlation times for the probes in this system lie in the borderline range for which there is no unique calibration in terms either of $T_{1}$ or of the $\left(T_{1} T_{2}\right)$ product. For this reason, the spin packet widths (or intrinsic $T_{2}$ ) were determined from lineshape simulations of the conventional EPR spectra. These values and the $T_{1}$-values derived from calibration curves such as those of Fig. 5 are given in Table 4 .

The relaxation enhancement induced by addition of the paramagnetic salt $\mathrm{NiCl}_{2}$ in the aqueous phase was also studied for TEMPO-stearate in DOPC bilayers. The $\left(T_{1} T_{2}\right)$ products that were obtained by using calibrations such as those in Fig. 4 were found to decrease with increasing $\mathrm{Ni}^{2+}$ ion concentration. Because this probe is exposed to water one would expect strong Heisenberg spin exchange at high concentrations of aqueous $\mathrm{Ni}^{2+}$ ions that will make equal contributions to the $T_{1^{-}}$ and $T_{2}$-relaxation rates of the TEMPO-stearate spin label. By using the latter condition, the spin-lattice relaxation time, $T_{1}$, can be extracted readily from the measured values of $\left(T_{1} T_{2}\right)$. The resulting values of the spin-lattice relaxation rate, $1 / T_{1}$, were found to increase essentially linearly with $\mathrm{Ni}^{2+}$ ion concentration, as expected for a true relaxation enhancement by Heisenberg exchange (see Table 4).

3. Lipid spin label in gel-phase lipid bilayers. The saturation transfer EPR spectra of the 5-PCSL spin label in DPPC bilayers in the gel phase at $15^{\circ} \mathrm{C}$ were characteristic of an effective rotational correlation time in the region of $10^{-5} \mathrm{~s}$ (see, e.g., Ref. 24). This corresponds to a slow rotational mobility for which the $\left(T_{1} T_{2}\right)$ relaxation time product may be determined from the saturation curves. These values deduced from the appropriate calibrations are given in Table 5. Lineshape analysis of the low-field spectral component with Gaussian-Lorentzian convolution indicated that the Lorentzian component dominated and essentially accounted for the whole of the half-width values. The values of $T_{2}$ deduced in this way

TABLE 5

Influence of $\mathrm{Ni}^{2+}$ Ion Concentration on the R elaxation Parameters Deduced from the Saturation Properties and Linewidths of 5-PCSL in DPPC Bilayers in the Gel Phase at $15^{\circ} \mathrm{C}$

\begin{tabular}{|c|c|c|c|c|}
\hline$\left[\mathrm{Ni}^{2+}\right](\mathrm{mM})$ & $\frac{10^{-14}}{\left(T_{1} T_{2}\right)^{e f f}}\left(\mathrm{~s}^{-2}\right)$ & $\begin{array}{c}\left(T_{1} T_{2}\right) \times 10^{14} \\
\left(\mathrm{~s}^{2}\right)\end{array}$ & $T_{2}(\mathrm{~ns})^{a}$ & $T_{1}(\mu \mathrm{s})$ \\
\hline 0 & $0.31 \pm 0.01$ & $3.2 \pm 0.15$ & $19 \pm 0.7$ & $1.7 \pm 0.1$ \\
\hline 5 & $0.49 \pm 0.02$ & $2.1 \pm 0.1$ & $19 \pm 0.7$ & $1.1 \pm 0.1$ \\
\hline 10 & $0.495 \pm 0.025$ & $2.0 \pm 0.1$ & $21 \pm 1$ & $0.95 \pm 0.1$ \\
\hline 15 & $0.61 \pm 0.02$ & $1.65 \pm 0.07$ & $18 \pm 0.8$ & $0.9 \pm 0.08$ \\
\hline 20 & $0.7 \pm 0.02$ & $1.45 \pm 0.05$ & $18 \pm 0.8$ & $0.8 \pm 0.07$ \\
\hline 30 & $0.90 \pm 0.03$ & $1.1 \pm 0.04$ & $17 \pm 0.6$ & $0.65 \pm 0.05$ \\
\hline 40 & $1.2 \pm 0.04$ & $0.87 \pm 0.04$ & $20 \pm 1$ & $0.44 \pm 0.04$ \\
\hline $5+60 \mathrm{mM} \mathrm{CaCl}_{2}$ & $0.32 \pm 0.01$ & $3.15 \pm 0.15$ & $17 \pm 0.6$ & $1.8 \pm 0.1$ \\
\hline $10+60 \mathrm{mM} \mathrm{CaCl}_{2}$ & $0.32 \pm 0.01$ & $3.15 \pm 0.15$ & $17 \pm 0.6$ & $1.8 \pm 0.1$ \\
\hline $30+60 \mathrm{mM} \mathrm{CaCl}_{2}$ & $0.44 \pm 0.02$ & $2.3 \pm 0.12$ & $23 \pm 1.5$ & $1.0 \pm 0.1$ \\
\hline
\end{tabular}

${ }^{a}$ Deduced from the linewidth of the low-field extremum in the quasi-powder spectrum (see text). 


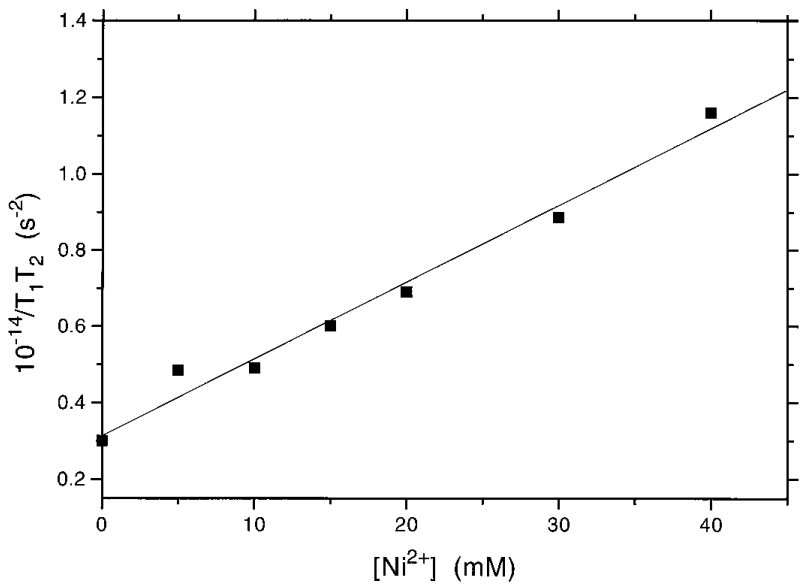

FIG. 7. Dependence of the reciprocal relaxation time product, $1 /\left(T_{1} T_{2}\right)$, deduced from calibrations of the saturation curves, on $\mathrm{Ni}^{2+}$ ion concentration in the aqueous medium for the 5-PCSL spin label in gel-phase DPPC bilayers at $15^{\circ} \mathrm{C}$.

$\left(\Delta H=1 / \gamma_{e} T_{2}\right)$ were independent of $\mathrm{Ni}^{2+}$ ion concentration in the aqueous phase (see Table 5) and were used to provide estimates of the $T_{1}$ relaxation times from the $\left(T_{1} T_{2}\right)$ products obtained in the saturation studies. These values are also given in Table 5. The dependences of $1 /\left(T_{1} T_{2}\right)$ and of $1 / T_{1}$ on $\mathrm{Ni}^{2+}$ ion concentration were again linear (see Fig. 7), as expected for a spin-lattice relaxation enhancement by dipole-dipole interactions with the fast-relaxing $\mathrm{Ni}^{2+}$ species. In the presence of excess $\mathrm{Ca}^{2+}$ ions, added $\mathrm{Ni}^{2+}$ was found to have little effect on the $T_{1}$ relaxation times (Table 5). This, together with the lack of influence of $60 \mathrm{mM} \mathrm{CaCl}{ }_{2}$ on the $T_{1}$-relaxation, confirms that the relaxation enhancement induced by $\mathrm{Ni}^{2+}$ in the absence of $\mathrm{Ca}^{2+}$ is a paramagnetic (dipolar) effect. The concentration of divalent ion binding sites at the bilayer surface was 5-10 times larger than the ion concentrations used. Therefore, adsorption of $\mathrm{Ca}^{2+}$ at the lipid surface depleted the surface regions of $\mathrm{Ni}^{2+}$ ions in a noncompetitive manner because of the repulsive electrostatic potential built up at the bilayer surface.

\section{DISCUSSION}

The saturation curves for the second integral of the first harmonic in-phase absorption spectra of nitroxide spin labels take the form predicted by simple slow-passage conditions in the absence of molecular motion. This is true for all likely experimental conditions, including high modulation frequencies and the presence of both molecular reorientation and inhomogeneous broadening. These results have been established by spectral simulations that take explicit account of the microwave and modulation fields, as well as using models for the molecular motion that cover all accessible correlation time regimes. Analyzing saturation curves by means of the double integrated intensity of the conventional
EPR spectrum is therefore appropriate not only in allowing for inhomogeneous broadening, as suggested previously (7), but also in comparing systems with different mobilities. In the latter case, however, calibrations appropriate to the particular rotational correlation time (and modulation frequency) must be used to extract the true relaxation times. These calibrations have been established here by means of the spectral simulations. Because ${ }^{14} \mathrm{~N}$ nuclear relaxation is not considered explicitly in the theoretical treatment, the spin-lattice relaxation times deduced are effective values that include the effects of nuclear relaxation. This point is covered in detail in the Appendix. The assumption of equal effective electron relaxation times for all three hyperfine lines is reasonable in the presence of nuclear relaxation, and, over a wide range of rotational correlation times, the intrinsic electron relaxation time is three times the effective value (see Appendix).

An important result found from the dependence of the saturation properties on molecular reorientation rate is that, for the intermediate regime $\left(2 \times 10^{7} \mathrm{~s}^{-1}<f_{R}<2 \times 10^{8}\right.$ $\mathrm{s}^{-1}$ ), the saturation properties depend only weakly on the intrinsic $T_{2}^{0}$. Values of $T_{1}$ may then be estimated directly from the saturation curves, rather than values of the $\left(T_{1} T_{2}\right)$ product that normally are extracted from progressive saturation experiments. This intermediate regime is readily identified from the extreme motional broadening of the spectra that also provides a sensitive means for determining the molecular reorientation rate from the lineshapes.

The methods have been applied to experimental systems, including those of biological interest, which are representative of the different rotational correlation time regimes. These include both the fast and slow regimes where the $\left(T_{1} T_{2}\right)$ relaxation time product may be determined, as well as the intermediate regime for which a direct determination of $T_{1}$ is possible. The experimental validity of the method has been established from the linearity of the measured increase in spin-lattice relaxation rate with increasing $\mathrm{Ni}^{2+}$ ion concentration in the aqueous phase, for two spin-labeled membrane systems. The latter correspond to true relaxation enhancements induced both by Heisenberg spin exchange with the surface-exposed TEMPO-stearate spin label and by magnetic dipole-dipole interactions with the membraneembedded 5-PCSL spin label. These illustrate two common applications of this branch of spin-label methodology, namely in determining accessibility of spin-labeled groups (e.g., Refs. 1, 25) and in measuring the depth of spin-labeled groups in membranes (26).

Of considerable interest are the significantly longer $T_{1^{-}}$ relaxation times obtained for TEMPOL in glycerol solutions, as compared with the lipid spin labels in micelles, fluid phospholipid bilayers, and even gel-phase bilayers (cf. Tables 3-5). For 5-PCSL in lysoPC micelles, and 14-PCSL and TEMPO-stearate in fluid DOPC bilayers, the effective correlation times (for overall motion) are close to that of 
TEMPOL in $90 \%$ glycerol. In the case of 5-PCSL in gelphase DPPC, the effective correlation time for overall motion is much longer. The origin of these differences in $T_{1}$ most probably arises, at least in part, from Heisenberg spin exchange because of the higher effective (i.e., two-dimensional) spin label concentrations in the lipid systems. An additional possibility lies in the fact that $T_{1}$-relaxation is determined by fast, restricted local motions, which have appreciable spectral densities at the Larmor frequency. These motions are known to exist in fluid-phase membranes, for example, from trans-gauche isomerism (27). In gelphase membranes, they are probably of librational character and are responsible for the practically homogeneous lineshape of 5-PCSL in DPPC at $15^{\circ} \mathrm{C}$. Such fast local motions are presumably absent for the small spin label TEMPOL, or are damped in the viscous glycerol medium, resulting in a decreased spin-lattice relaxation rate.

When allowance is made for nuclear relaxation (see Appendix), the effective $T_{1}$-relaxation times obtained here for 14-PCSL in fluid-phase DOPC at $20^{\circ} \mathrm{C}$ and for 5-PCSL in gel-phase DPPC at $15^{\circ} \mathrm{C}$ yield corrected values of $T_{1 e} \sim 1.5$ and $5 \mu$ s, respectively. These may be compared with values for the intrinsic $T_{1}$-relaxation time that have been obtained from saturation recovery EPR measurements. In the fluid phase these are $T_{1 e}=2.25 \mu$ s for 16-C atom labeled stearic acid (16-SASL) in dimyristoyl phosphatidylcholine (DMPC) at $27^{\circ} \mathrm{C}(28)$ and $T_{1 e}=1.6 \mu \mathrm{s}$ in DOPC at $54^{\circ} \mathrm{C}$ (29). In the gel phase, $T_{1 e}=8.5 \mu \mathrm{s}$ has been obtained by saturation recovery for 6-PCSL in DPPC at $1{ }^{\circ} \mathrm{C}(30)$ and $T_{1 e}$ $=8 \mu \mathrm{s}$ for 5 -SASL in DMPC at $4^{\circ} \mathrm{C}(31)$. It is seen that the two methods yield $T_{1 e^{-v a l u e s}}$ of a similar magnitude in comparable systems, and in particular, reflect the difference in rotational correlation time between fluid- and gel-phase membranes in the same way. Reasons for residual differences may lie in the different conditions, including temperatures and spin-label concentrations, employed in the two sets of experiments, as well as the different ways that nuclear relaxation was handled in the various measurements.

Future developments of the method that are envisaged include the extension to other motional models, particularly to anisotropic rotation. The general formulation given in the Theory section allows immediate evaluation of the nonlinear spectra that are detected out-of-phase with respect to the field modulation, including dispersion components. Extension to include higher harmonics is also possible in a straightforward manner. These latter will generally allow exploration of nonlinear EPR displays that may have enhanced sensitivity to spin-lattice relaxation. For the out-of-phase second harmonic absorption (saturation transfer EPR) spectra, feasibility has already been demonstrated experimentally (see, e.g., Refs. $4,26)$.

\section{APPENDIX: NUCLEAR RELAXATION}

The simulations given for the saturation behavior of the spin label EPR spectrum have taken into account molecular motion and the microwave $H_{1}$ and Zeeman modulation fields, but nuclear relaxation between the hyperfine levels of the ${ }^{14} \mathrm{~N}$ nitroxide has been neglected. Therefore, the spin-lattice relaxation times that are obtained from the calibration of the progressive saturation experiments are effective ones, $T_{1}^{e f f}$, that contain contributions from ${ }^{14} \mathrm{~N}$-nuclear relaxation. The dependence of the effective $T_{1}$-relaxation time on nuclear magnetic quantum number, $m_{I}$, is, however, relatively small. The dependence of $T_{1}^{\text {eff }}$ on nuclear relaxation rate for the $m_{I}=0$ and $m_{I}$ $= \pm 1{ }^{14} \mathrm{~N}$-hyperfine manifolds is given, respectively, by (see, e.g., Ref. 32)

$$
\begin{aligned}
T_{1}^{e f f}(0) & =\left(\frac{1+b}{1+3 b}\right) T_{1 e} \\
T_{1}^{e f f}( \pm 1) & =\frac{1+3 b+b^{2}}{(1+b)(1+3 b)} \cdot T_{1 e},
\end{aligned}
$$

where $b=T_{1 e} / T_{1 n}$ is the ratio of the nuclear to the electron spin-lattice relaxation rates, and $T_{1 e}\left(T_{1 n}\right)$ is the intrinsic electron (nuclear) spin-lattice relaxation time. Saturation recovery experiments have shown that the intrinsic $T_{1 e}$ does not depend on nuclear magnetic quantum number (see, e.g., Ref. 22 for a summary). The dependence on nuclear relaxation rate of the difference in $T_{1}^{e f f}$ between the $m_{I}=0$ and $m_{I}= \pm 1{ }^{14} \mathrm{~N}$ hyperfine manifolds is shown in Fig. A.1 (solid line). The maximum difference is $25 \%$, which is obtained for $T_{1 n}=T_{1 e}$, and this difference decreases to zero for very fast or very slow nuclear relaxation. The assumption made in fitting the saturation curves, that the effective $T_{1}$ is the same for all three hyperfine lines, is therefore a reasonable approximation in the presence of nuclear relaxation.

As seen from Fig. A.1, the effective spin-lattice relaxation time, $T_{1}^{\text {eff }}(0)$, does, however, depend on the nuclear relaxation time (dashed line). For $T_{1 n}=T_{1 e}$, the effective relaxation time is $T_{1}^{e f f}(0)=\frac{1}{2} T_{1 e}$, and for very fast nuclear relaxation the effective electron relaxation time tends to a limiting value of $T_{1}^{\text {eff }}(0)=\mathrm{T}_{1}^{\text {eff }}( \pm 1)=(1 / 3) \mathrm{T}_{1 \mathrm{e}}$. Knowledge of the nuclear relaxation rate is therefore required to obtain the intrinsic electron spin-lattice relaxation time from progressive saturation EPR measurements. The database of $T_{1 n}$-values for the normal ${ }^{14} \mathrm{~N}$-nitroxide spin labels is currently rather small. However, it does appear, except for very short rotational correlation times, that $T_{1 n} \leq T_{1 e}$, i.e., $b \geq 1$ (see, e.g., Ref. 22). Therefore, $T_{1 e}$ is likely to lie within the range $2 T_{1}^{\text {eff }}(0)$ to $3 T_{1}^{\text {eff }}(0)$, with the precise value depending on the nuclear relaxation rate and hence on the rotational correlation time. The dependence of the normalized nuclear relaxation rate, $b=$ $T_{1 e} / T_{1 n}$, on the rotational correlation time, $\tau_{R}$, of the spin label is given in Fig. A.2. (solid lines). Although it is for a ${ }^{15} \mathrm{~N}$ - 
nitroxide spin label, this data, which is calculated from the results of Robinson et al. (33), does give some idea of the likely values of $b$ that may be used to obtain a reasonable estimate of the intrinsic electron spin-lattice relaxation time from the effective values that are obtained from progressive EPR experiments. The dependence on rotational correlation time of the ratio, $T_{1}^{e f f}(0) / T_{1 e}$, of the effective electron relaxation time to the intrinsic relaxation time that is predicted from Eq. [A.1] for a ${ }^{14} \mathrm{~N}$-nitroxide spin label with the same values of $b$ is also given in Fig. A.2 (dashed line). Over a rather wide range of rotational correlation times from $\tau_{R} \sim 5 \times 10^{-10} \mathrm{~s}$ to $\tau_{R} \sim 5 \times 10^{-7} \mathrm{~s}$, the effective relaxation time remains approximately constant at $T_{1}^{e f f}(0) \approx(1 / 3) T_{1 e}$ (see Fig. A.2, dashed line). At very long correlation times, $\tau_{R} \geq 10^{-4} \mathrm{~s}$, the effective relaxation time levels off at a value of $T_{1}^{e f f}(0)=0.39$ $\times T_{1 e}$, and at very short correlation times, $\tau_{R}<10^{-10} \mathrm{~s}$, the effective relaxation time increases steadily to a limiting value of $T_{1}^{e f f}(0)=T_{1 e}$.

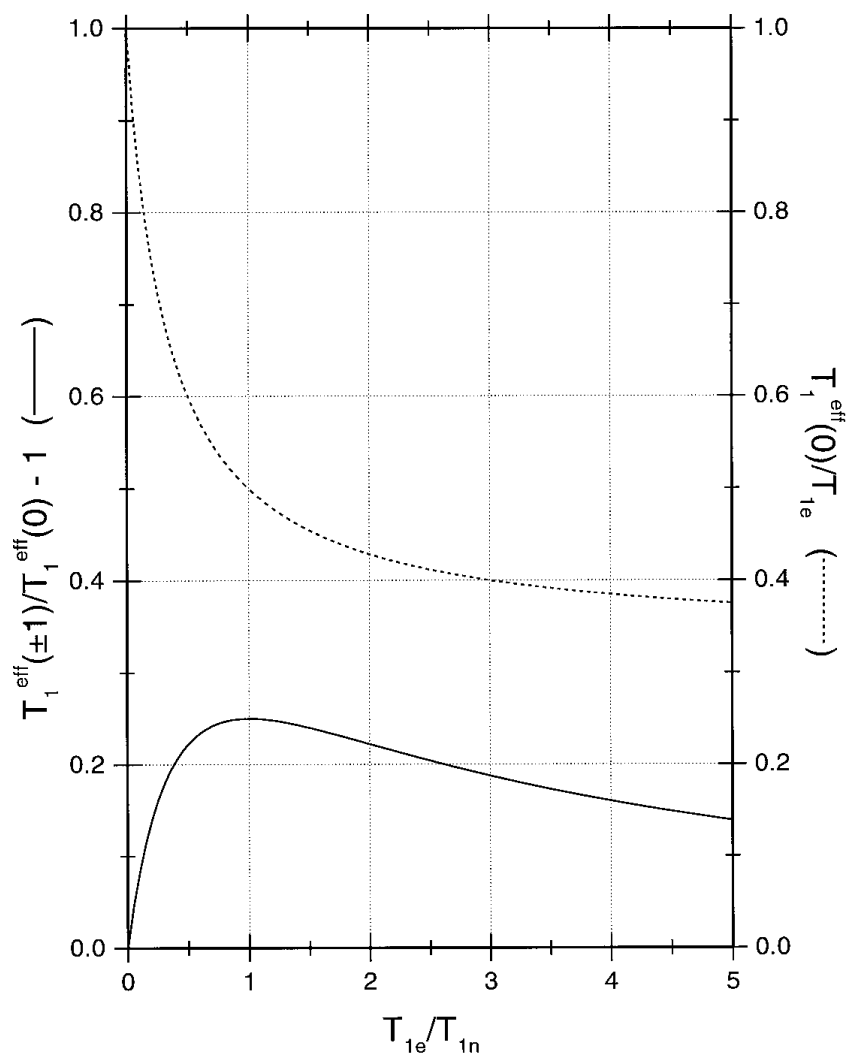

FIG. A.1. Dependence on the ratio of nuclear to electron spin-lattice rates ( $b=T_{1 e} / T_{1 n}$ ) of (solid line) the normalized difference in effective spinlattice relaxation times, $T_{1}^{\text {eff }}( \pm 1) / T_{1}^{\text {eff }}(0)-1$, of the $m_{I}= \pm 1$ and $m_{I}=0$ hyperfine manifolds, and of (dashed line) the ratio, $T_{1}^{e f f}(0) / T_{1 e}$, of the effective $T_{1}$ of the $m_{I}=0$ manifold to the intrinsic electron $T_{1}$. The dependences are calculated from Eqs. [A.1] and [A.2].

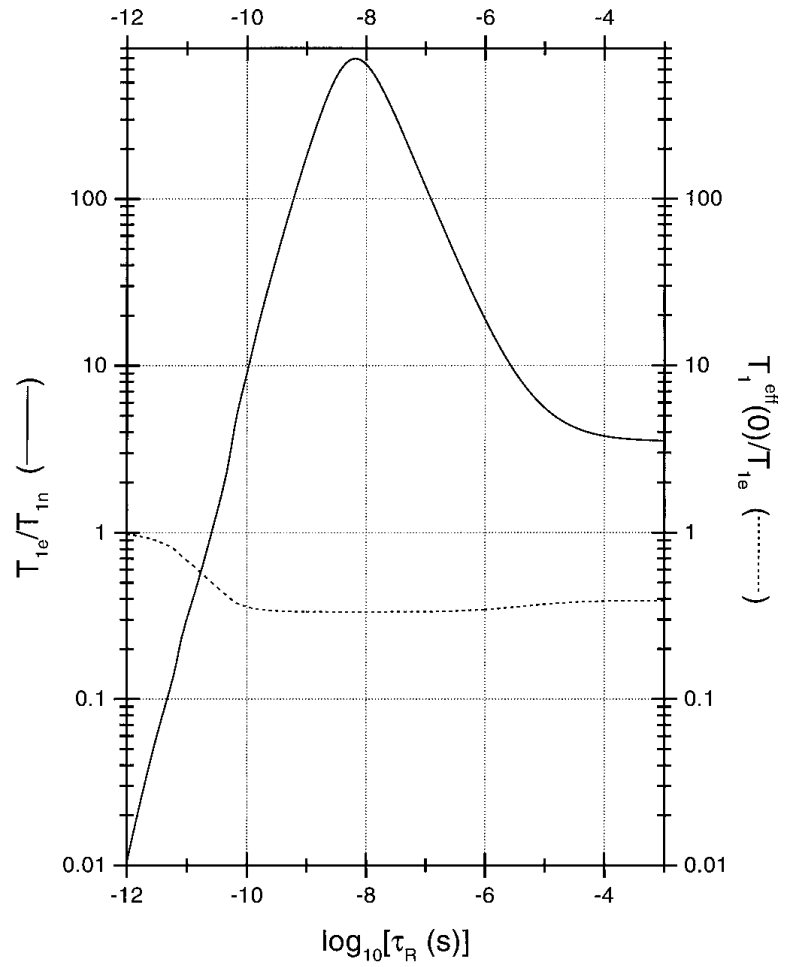

FIG. A.2. Dependence of the nuclear relaxation rate, $b=T_{1 e} / T_{1 n}$ (solid line), on the rotational correlation time $\left(\log _{10} \tau_{R}\right)$ of the spin label. The dependence is calculated from fits to experimental data on the perdeuterated ${ }^{15} \mathrm{~N}$-TEMPOL spin label in glycerol-water mixtures that are given in Ref. (33). Oxygen-induced relaxation is neglected. The values of the effective spinlattice relaxation time of the $m_{I}=0$ hyperfine manifold, $T_{1}^{e f f}(0) / T_{1 e}$, for a ${ }^{14} \mathrm{~N}$-nitroxide spin label with the same values of $b$ are given from Eq. [A.1] by the dashed line.

\section{ACK NOWLEDGMENTS}

This work was supported partly by the Deutsche Forschungsgemeinschaft. The work of V. A. Livshits was supported in part by the Russian Foundation for Basic Research, grant 95-04-09604, and that of T. Páli in part by the Hungarian National Science Foundation F5506/1992. We gratefully thank Dr. Boris Dzikovski for his kind assistance with some of the experiments.

\section{REFERENCES}

1. C. Altenbach, T. Marti, H. G. Khorana, and W. L. Hubbell, Transmembrane protein structure: spin labeling of bacteriorhodopsin mutants, Science 248, 1088-1092 (1990).

2. W. L. Hubbell and C. Altenbach, Site-directed spin-labeling of membrane proteins, in 'Membrane Protein Structure. Experimental Approaches' (S. H. White, Ed.), pp. 224-248, Oxford University Press, New York, Oxford (1994).

3. M. M. E. Snel and D. Marsh, Accessibility of spin-labeled phospholipids in anionic and zwitterionic bilayer membranes to paramagnetic relaxation agents. Continuous wave power saturation EPR studies, Biochim. Biophys. Acta 1150, 155-161 (1993).

4. L. I. Horváth, P. J. Brophy, and D. Marsh, Exchange rates at the lipid-protein interface of the myelin proteolipid protein determined by saturation transfer electron spin resonance and continuous wave saturation studies, Biophys. J . 64, 622-631 (1993). 
5. D. Marsh, Exchange and dipolar spin-spin interactions and rotational diffusion in saturation transfer EPR spectroscopy, Appl. Magn. Reson. 3, 53- 65 (1992).

6. D. Marsh, Progressive saturation and saturation transfer ESR for measuring exchange processes of spin-labelled lipids and proteins in membranes, Chem. Soc. Rev. 22, 329-335 (1993).

7. T. Páli, L. I. Horváth, and D. Marsh, Continuous-wave saturation of two-component, inhomogeneously broadened, anisotropic EPR spectra, J. Magn. Reson. A 101, 215-219 (1993).

8. D. Haas, C. Mailer, and B. H. Robinson, Using nitroxide spin labels. How to obtain $\mathrm{T}_{1 \mathrm{e}}$ from continuous wave electron paramagnetic resonance spectra at all rotational rates, Biophys. J . 64, 594-604 (1993).

9. V. A. Livshits, Slow anisotropic tumbling in ESR spectra of nitroxyl radicals, J. Magn. Reson. 24, 307-313 (1976).

10. V. A. Livshits, The application of microwave saturation for studying slow tumbling of nitroxyl radicals, Theor. i Exper. Khim. 13, 363370 (1977).

11. V. A. Livshits and Yu. A. Bobrov, Anisotropy of spin probe molecular rotation as studied by saturation transfer ESR spectroscopy, Theor. i Exper. Khim. 22, 331-336 (1986).

12. D. Marsh and A. Watts, Spin-labeling and lipid-protein interactions in membranes, in 'Lipid-Protein Interactions' (P. C. J ost and O. H. Griffith, Eds.), pp. 53-126, Wiley-Interscience, New York (1982).

13. P. Fajer and D. Marsh, Microwave and modulation field inhomogeneities and the effect of cavity Q in saturation transfer ESR spectra. Dependence on sample size, J. Magn. Reson. 49, 212-224 (1982).

14. M. A. Hemminga, P. A. de J aeger, D. Marsh, and P. Fajer, Standard conditions for the measurement of saturation transfer ESR spectra, J. Magn. Reson. 59, 160-163 (1984).

15. K. Halbach, Über eine neue Methode zur Messung von Relaxationszeiten und über den Spin von $\mathrm{Cr}^{53}$, Helv. Phys. Acta 27, 259-282 (1954).

16. W. A. Anderson, Nuclear magnetic resonance spectra of some hydrocarbons, Phys. Rev. 102, 151-167 (1956).

17. R. C. McCalley, E. J. Shimshick, and H. M. McConnell, The effect of slow rotational motion on paramagnetic resonance spectra, Chem. Phys. Lett. 13, 115-119 (1972).

18. S. A. Goldman, G. V. Bruno, C. F. Polnaszek, and J. H. Freed, An ESR study of anisotropic rotational reorientation and slow tumbling in liquid and frozen media, J. Chem. Phys. 56, 716-735 (1972).

19. J. Israelachvili, J. Sjösten, L. E. G. Eriksson, M. Ehrström, A. Gräslund, and A. Ehrenberg, ESR spectral analysis of the molecular motion of spin labels in lipid bilayers and membranes based on a model in terms of two angular motional parameters and rotational correlation times, Biochim. Biophys. Acta 382, 125-141 (1975).

20. J. S. Hyde and L. R. Dalton, Saturation-transfer spectroscopy, in 'Spin-Labeling II. Theory and Applications' (L. J . Berliner, Ed.), pp. 1-70, Academic Press, New York (1979).

21. T. C. Squier and D. D. Thomas, Selective detection of the rotational dynamics of the protein-associated lipid hydrocarbon chains in sarcoplasmic reticulum membranes, Biophys. J. 56, 735-748 (1989).

22. D. Marsh, T. Páli, and L. I. Horváth, Progressive saturation and saturation transfer EPR for measuring exchange processes and proximity relations in membranes, in 'Spin Labeling: The Next Millenium' (L. J . Berliner, Ed.), in press, Plenum, New York (1998).

23. J. H. Freed, Theory of slow tumbling ESR spectra for nitroxides, in 'Spin Labeling. Theory and Applications' (L. J . Berliner, Ed.), pp. 53-132, Academic Press, New York (1976).

24. L. I. Horváth and D. Marsh, Improved numerical evaluation of saturation transfer electron spin resonance spectra, J. Magn. Reson. 80, 314-317 (1988).

25. M. M. E. Snel, B. de Kruijff, and D. Marsh, Membrane location of spin-labeled apocytochrome $\mathrm{c}$ and cytochrome $\mathrm{c}$ determined by paramagnetic relaxation agents, Biochemistry 33, 11150-11157 (1994).

26. T. Páli, R. Bartucci, L. I. Horváth, and D. Marsh, Distance measurements using paramagnetic ion-induced relaxation in the saturation transfer electron spin resonance of spin-labeled biomolecules. Application to phospholipid bilayers and interdigitated gel phases, Biophys. J . 61, 1595-1602 (1992).

27. M. Moser, D. Marsh, P. Meier, K.-H. Wassmer, and G. Kothe, Chain configuration and flexibility gradient in phospholipid membranes. Comparison between spin-label electron spin resonance and deuteron nuclear magnetic resonance, and identification of new conformations, Biophys. J . 55, 111-123 (1989).

28. C. A. Popp and J. S. Hyde, Electron-electron double resonance and saturation recovery studies of nitroxide electron and nuclear spin-lattice relaxation times and Heisenberg exchange rates: lateral diffusion in dimyristoylphosphatidylcholine, Proc. Natl. Acad. Sci. USA 79, 2559-2563 (1982).

29. J.J . Yin, M. Pasenkiewicz-Gierula, and J . S. Hyde, Lateral diffusion of lipids in membranes by pulse saturation recovery electron spin resonance, Proc. Natl. Acad. Sci. USA 84, 964-968 (1987).

30. P. Fajer, A. Watts, and D. Marsh, Saturation transfer, continuous wave saturation, and saturation recovery electron spin resonance studies of chain-spin labeled phosphatidylcholines in the low temperature phases of dipalmitoyl phosphatidylcholine bilayers. Effects of rotational dynamics and spin-spin interactions, Biophys. J . 61, 879-891 (1992).

31. A. Kusumi, W. K. Subczynski, and J. S. Hyde, Oxygen transport parameter in membranes as deduced by saturation recovery measurements of spin-lattice relaxation times of spin labels, Proc. Natl. Acad. Sci. USA 79, 1854-1858 (1982).

32. D. Marsh, Influence of nuclear relaxation on the measurement of exchange frequencies in CW saturation EPR studies, J. Magn. Reson. 99, 332-337 (1992).

33. B. H. Robinson, D. A. Haas, and C. Mailer, Molecular dynamics in liquids: Spin-lattice relaxation of nitroxide spin labels, Science 263, $490-493$ (1994). 